\title{
Philosophiques
}

\section{Kafka et Brentano}

\section{Barry Smith}

Volume 26, numéro 2, automne 1999

La critique de la raison en Europe centrale

URI : https://id.erudit.org/iderudit/004986ar

DOI : https://doi.org/10.7202/004986ar

Aller au sommaire du numéro

\section{Éditeur(s)}

Société de philosophie du Québec

\section{ISSN}

0316-2923 (imprimé)

1492-1391 (numérique)

Découvrir la revue

\section{Citer cet article}

Smith, B. (1999). Kafka et Brentano. Philosophiques, 26(2), 349-371.

https://doi.org/10.7202/004986ar

\section{Résumé de l'article}

Un mince fil dans la vaste littérature sur Kafka concerne la connaissance qu'avait Kafka de la philosophie, et plus précisément l'utilisation, dans les récits de Kafka, de quelques-unes des idées principales de Franz Brentano. Kafka a suivi des cours de philosophie à l’Université Charles, cours donnés par des étudiants de Brentano, Anton Marty et Christian von Ehrenfels. Il fut aussi, pendant plusieurs années, membre d'un groupe de discussion organisé par des partisans orthodoxes de la philosophie brentanienne à Prague. Le présent essai résume ce que l'on sait des relations entretenues par Kafka avec le mouvement brentanien. Il porte sur les idées brentaniennes qui concernent l'évidence de la perception intérieure, la conscience oblique, l'introspection active, le jugement correct et incorrect et la conscience en tant qu'espèce de tribunal intérieur, dans le but d'éclairer certains aspects centraux de l'œuvre de Kafka, incluant son style. Nous porterons une attention particulière sur Die Verwandlung et Der Prozess, et nous offrirons une interprétation de ce dernier texte selon laquelle le procès de Joseph K. se passe entièrement dans l'esprit de K. lui-même.
Ce document est protégé par la loi sur le droit d'auteur. L’utilisation des services d’Érudit (y compris la reproduction) est assujettie à sa politique d'utilisation que vous pouvez consulter en ligne.

https://apropos.erudit.org/fr/usagers/politique-dutilisation/ 


\title{
Kafka et Brentano
}

\author{
BARRY SMITH \\ University of Buffalo \\ Phismith@acsu.buffalo.edu
}

\begin{abstract}
RÉSUMÉ. - Un mince fil dans la vaste littérature sur Kafka concerne la connaissance qu'avait Kafka de la philosophie, et plus précisément l'utilisation, dans les récits de Kafka, de quelques-unes des idées principales de Franz Brentano. Kafka a suivi des cours de philosophie à l'Université Charles, cours donnés par des étudiants de Brentano, Anton Marty et Christian von Ehrenfels. Il fut aussi, pendant plusieurs années, membre d'un groupe de discussion organisé par des partisans orthodoxes de la philosophie brentanienne à Prague. Le présent essai résume ce que l'on sait des relations entretenues par Kafka avec le mouvement brentanien. Il porte sur les idées brentaniennes qui concernent l'évidence de la perception intérieure, la conscience oblique, l'introspection active, le jugement correct et incorrect et la conscience en tant qu'espèce de tribunal intérieur, dans le but d'éclairer certains aspects centraux de l'œuvre de Kafka, incluant son style. Nous porterons une attention particulière sur Die Verwandlung et Der Prozess, et nous offrirons une interprétation de ce dernier texte selon laquelle le procès de Joseph K. se passe entièrement dans l'esprit de K. lui-même.
\end{abstract}

ABSTRACT. - There is a narrow thread in the vast literature on Kafka which pertains to Kafka's knowledge of philosophy, and more precisely to Kafka's use in his fictional writings of some of the main ideas of Franz Brentano. Kafka attended courses in philosophy at the Charles University given by Brentano's students Anton Marty and Christian von Ehrenfels, and was for several years a member of a discussion-group organized by orthodox adherents of the Brentanian philosophy in Prague. The present essay summarizes what is known about Kafka's relations to the Brentanist movement. It draws on Brentanian ideas on the evidence of inner perception, on oblique consciousness, on active introspection, on correct and incorrect judgment, and on consciousness as a species of inner tribunal, in order to throw light on central features of Kafka's writings, including stylistic features. Special attention is directed towards Die Verwandlung and Der Prozess, and a reading of the latter is offered according to which the trial of Joseph K. occurs entirely within the mind of K. himself.

[N ]ous sommes comme des troncs d'arbres dans la neige. En apparence, ils reposent moelleusement et un petit coup serait suffisant pour les faire rouler. $\mathrm{N}$ on, cela ne peut se faire, puisqu'ils sont fermement unis au sol. M ais regardez, même ceci n'est qu'apparence. ${ }^{1}$

Un mince fil dans la vaste littérature sur K afka se rapporte à la connaissance qu'avait Kafka de la philosophie et plus précisément à la fécondité des ten-

1. «Die Baüme», Kafka, 1970, p. 19 (traduction anglaise de W. et E. M uir, Kafka, 1971, p. 382). 
tatives d'interpréter les écrits de Kafka à la lumière des idées principales de Franz Brentano. De telles tentatives ont été carrément écartées, entre autres par $M$ ax Brod, qui a nié le rôle de toute théorie dans les écrits de Kafka (Kafka parlait en images parce qu'il pensait en images). Comme A rnold $\mathrm{H}$ eidsieck l'a minutieusement documenté dans sa récente étude du contexte intellectuel de l'œuvre de K afka, les œuvres de fiction de Kafka sont bien informées des débats publics et académiques au cours de la première décade du vingtième siècle sur la physiologie, la psychologie cognitive et linguistique, la philosophie de l'esprit et du langage ; la loi positive et la théorie de la loi naturelle; les procédures criminelles, l'éthique et la religion?2.

Kafka s'est instruit et impliqué jusqu'à un certain niveau dans ces débats, entre autres par la fréquentation de ses collègues étudiants (dont Hugo Bergmann et Emil Utitz), par les cours de philosophie auxquels il a assisté à I'U niversité C harles de Prague (des cours donnés entre autres par les étudiants de Brentano, Christian von Ehrenfels et Anton M arty), et par son adhésion durant trois années à un groupe de discussion organisé par des adhérents orthodoxes de la philosophie brentanienne de Prague. Le livre de $\mathrm{H}$ eidsieck fait le survol des théorèmes philosophiques qui sont ou bien intensément satisfaisants ou bien inadéquats selon les brentaniens de Prague, et il tente de montrer comment « $\mathrm{K}$ afka embeds them almost serially in his developing themes and paradigms $»^{3}$. Ici, je me concentrerai sur deux de ces « théorèmes philosophiques »: la doctrine brentanienne de l'intentionnalité et le compte-rendu du jugement éthique chez Brentano.

Grâce au travail des historiens des idées des dernières décennies, nous commençons à comprendre quelque peu cette entité culturelle complexe qu'était le « fin de siècle » viennois. ${ }^{4}$ L'explosion d'énergie intellectuelle qui se rencontre dans les travaux de Brentano, $\mathrm{H}$ usserl, Weininger, Wittgenstein, Kraus, M ahler, Schœenberg, Loos, Klimt, H ofmannsthal, M usil, Broch, Schnitzler et Freud fut, pour une part, le produit de la très particulière fécondation croisée qui caractérisa la vie intellectuelle de l'A utriche. $M$ ais ce fut aussi, au moins en partie, le produit d'une plus large influence politique qui marqua la fin de l'empire des $\mathrm{H}$ absbourg. ${ }^{5}$ Les idées du libéralisme éclairé que soutinrent les intellectuels européens au cours du XVIII ${ }^{\mathrm{e}}$ et du XIX ${ }^{\mathrm{e}}$ siè-

2. Heidsieck 1994, p. 2 et suivantes.

3. Ibid., p. 48.

4. Consulter particulièrement les écrits de Carl E. Schorske, plus précisément ceux de 1960/1961 et de 1967 (réédition dans Schorske, 1980), et maintenant aussi N yiri, dir., 1981.

5. C'est en partie le reflet du caractère interdisciplinaire des études universitaires telles qu'elles s'étaient développées en Europe centrale, ce qu'illustre de manièrefrappantel'éducation de K afka. Sur le caractère encyclopédique du savoir autrichien, voir en général J ohnston, 1972, ch. 10 et suivants, et 19 et suivants, ainsi que Grassl et Smith, 1986. Ce n'est pas par hasard que I'unité du mouvement scientifique (cf. par exemple $\mathrm{N}$ eurath, 1938) naquit à Vienne. La phénoménologie peut aussi être considérée comme ayant été affectée par ce mouvement de pensée (cf. Küng, 1975). 
cles se révélèrent non viables ${ }^{6}$, en A utriche du moins, et l'effondrement de la foi libérale (et, plus généralement, de la foi en la politique comme moyen de perfectionnement de l'humanité) contribua à une introversion caractéristique des intellectuels autrichiens, à un éloignement dela société qui était aussi un rapprochement des régions plus profondes du soi isolé de l'individu.

Le présent essai est consacré non à Vienne mais à Prague, où les mêmes pressions politiques se faisaient sentir. II se penche plus spécialement sur I'œuvre de Franz Kafka et sur le mouvement brentanien de Prague avec lequel Kafka eut un bref contact dans sa jeunesse. Une introversion systématique est bien entendu évidente dans presque toutes les pages des écrits de K afka. On la sent toutefois aussi dans les travaux des disciples de Brentano pour lesquels la psychologie (science descriptive des structures de la conscience de l'individu) sert de discipline fondamentale fournissant des fondements théoriques définitifs non seulement à la logique et à l'éthique, par exemple, mais aussi aux disciplines que sont la politique et le droit. ${ }^{7}$

Kafka entre à I'U niversité allemande de Prague en novembre 1901. Ses cinq années d'études consistent en majeure partie en une fréquentation enthousiaste des cours de droit. II avait projeté à l'origine d'étudier la philosophie et un certain nombre de cours de philosophie étaient en fait obligatoires à l'époque pour les étudiants en droit. II semblerait donc que nous pouvons écarter la proposition suivant laquelle Kafka assistait à ces cours dans le seul but de satisfaire le règlement universitaire. $N$ ous avons des preuves convaincantes du fait que $K$ afka, pendant ce temps et durant un certain nombre d'années après, montra un intérêt prononcé pour la philosophie, et spécialement pour la philoso phie de Brentano ${ }^{8}$. II assista aussi à des cours de chimie $^{9}$, d'histoire du droit, de médecine légale, d'histoire de l'art et de l'architecture, de littérature allemande, d'économie et de science politique.

Les cours de philosophie auxquels K afka s'inscrivit au cours de la première année universitaire étaient les suivants :

- Semestre d'hiver 1901/02 : Ehrenfels: Philosophie pratique (4h/ semaine)

- Semestre d'été 1902 : Ehrenfels: Esthétique du drame M usical (1h/semaine)

- Semestre d'été 1902 : M arty : Problèmes fondamentaux de la psychologie descriptive (3h/semaine)

6. Cf. Schorske,1960/1961 et 1967, Smith, 1978, et, pour une défense particulièrement claire de la thèse de Schorske envers ses critiques (particulièrement J ohnston, 1972, et ailleurs), cf. N yiri, 1982.

7. Cf. Brentano, 1924, I.3, et 1934.

8. C'est certainement sous la pression de circonstances financières que $K$ afka décida finalement de passer sa licence de droit (cf. Wagenbach, 1958, spécialement p. 242 où la liste des membres de la classe terminale de K afka au Gymnase Allemand de Prague est reproduite).

9. I bid., spécialement p. 106-114 et N eesen, 1972. Pour un regard plus sceptique, voir Binder, 1966, p. 58 et suivantes, et 1980, vol. 1. 
Par «psychologie descriptive », M arty entendait la psychologie de son maître Brentano ${ }^{10}$, philoso phe et psychologue fortement influencé par A ristote et la scolastique ainsi que par Descartes, et qui exerça à son tour une large influence sur les intellectuels d'Europe centrale au cours des décennies qui marquèrent le tournant du siècle ${ }^{11}$. Ses élèves comprenaient, outre $M$ arty et Ehrenfels ${ }^{12}$, Freud, Thomas $M$ asaryk (qui fut, par la suite, le fondateur et premier président de la République de T chécoslovaquie), M einong, H usserl, Stumpf et Twardowski13.

Brentano lui-même fut maltraité par les autorités politiques viennoises. Ses étudiants furent toutefois capables d'obtenir des postes universitaires en A utriche et de propager la philosophie brentanienne au point qu'elle acquit le statut de philosophie semi-officielle de l'Empire. Les brentaniens les plus orthodoxes se réunissaient autour de $M$ arty à Prague, dont le cercle comprenait aussi d'anciens élèves de Brentano tels que $O$ skar K raus et $A$ Ifred $K$ astil. Les brentaniens praguois s'acquittèrent de la tâche de développer et de répandre les doctrines de Brentano avec une ferveur quasi religieuse. Un de leurs groupes se rencontrait régulièrement au C afé L ouvre sur la Ferdinandstrasse afin de « s'entraîner » aux modes de pensée brentaniens ainsi qu'à des débats et des disputes philosophiques. H ugo Bergmann, Emil Utitz et O skar Pollak, très proches compagnons d'école de $\mathrm{Kafka}^{14}$, étaient admis dans ce cercle, et Bergmann à son tour semblerait y avoir recruté Kafka. Des preuves attestent que, au moins entre les années 1903 et 1906, Kafka participa fréquemment aux réunions de ce qui était appelé « le cercle intime des brentaniens ${ }^{15}$.

10. Voir Brentano, 1982. Pour une transcription des leçons données par M arty sous ce titre, voir M arek et Smith, 1987.

11. On ne mettra pas seulement l'accent sur la relative ampleur de l'éducation universitaire d'Europe centrale, qui contribua au cosmopolitisme caractéristique des cités centre-européennes. II y a aussi d'importantes différences dans la manière dont la palette de disciplines était divisée en groupes de sujets, la relative prédominance du droit étant un facteur particulièrement important ici (cf. Smith, 1978).

12. J'ai parlé brièvement d'Ehrenfels - le père tutélaire de la G estalt Psychologie - en appendice d'une précédente version du présent essai (Smith, 1981). Ehrenfels fut pendant plusieurs années une figure familière à Prague. Voir, par exemple, le journal de Kafka du 4 février 1912 : "A musante scène quand le professeur Ehrenfels, qui devient de plus en plus élégant et qui - avec son crâne chauve qui dessine nettement à la lumière une courbe légèrement bombée en son sommet, avec sa voix de stentor qu'il module comme un instrument de musique, et un sourire confiant lors de la réunion - déclare qu'il est en faveur du mélange des races. » N ous savons qu'en 1913, Kafka assistait encore aux séminaires d'Ehrenfels (cf. son journal du 21 octobre 1913).

13. Sur l'influence de Brentano en général, voir H aller, 1978. Sur Brentano et Freud voir H eaton, 1981.

14. Bergmann fit plusieurs contributions importantes à la philosophie, particulièrement à celles de Bolzano et Brentano ; Utitz devint un esthéticien distingué, et Pollak un historien de I'art. C'est Pollak qui reçut et critiqua les premières expériences littéraires de Kafka (cf. Bezzel, 1975, Binder, 1980, et les références qui y sont données).

15. Wagenbach, 1958 ; N eesen, 1972 ; Binder, 1966, p. 58 et suivantes. 
Tel qu'Utitz le décrivit, le Cercle du Louvre était une communauté de penseurs :

Dans une lutte commune, nous nous rencontrions pour des soirées de discussion sans fin. Franz Brentano n'était, bien entendu, pas présent. $M$ ais sa puissante empreinte marquait n'importe quel de nos propos, que celui-ci exprimât une interprétation correcte de ses enseignements ou quelque objection à ceux-ci. ${ }^{16}$

$M$ ax Brod était aussi associé au Cercle du Louvre. N ous pensons qu'il commença à participer à ces réunions quelque temps après $\mathrm{K}$ afka, et probablement avant que leur amitié intime ne se fut établie. $M$ ais Brod ne semble jamais avoir vraiment appartenu au cercle intime des brentaniens, et il fut en fait cérémonieusement exclu de tous rapports avec le groupe après avoir publié dans une revue littéraire de Berlin une nouvelle qui comprenait une caricature du brentanisme religieux. II nous donna lui-même la description de son expulsion :

Quand j'entrai dans l'arrière-salle du Café Louvre, tout le monde était rassemblé dans une humeur bavarde et une copie de $G$ egenwart, qui était sorti peu avant la réunion, était posée sur la table. J'étais arrivé avec Kafka. Ce fut ÉmileU titz qui m'accusa en premier. [0 ]n en débattit quelque temps. Une série de délibérations suivirent, comme si ce qui se passait était une infraction au tribunal d'honneur estudiantin. Et personne ne prit ma défense, alors que tous les participants me connaissaient déjà (octobre 1905) depuis deux ans. Soudain l'un d'entre eux prit mon parti - Kafka. II était normalement si calme en société, donnant l'impression d'être presque apathique [...]. M ais alors Kafka me chuchota [...] qu'il vaudrait mieux que nous partions tous les deux et que nous abandonnions le cercle de Brentano pour toujours. Et nous le fîmes. ${ }^{17}$

Kafka, peu après cet événement, reprit toutefois contact avec quelques membres du Cercle du Louvre. D eux mois plus tard, il signa avec Pollak, Utitz et, entre autres, la belle-mère de Bergmann, Berta Fanta, la dédicace d'un livre de psychologie théorique ${ }^{18}$ qui fut offert à H ugo Bergmann à l'occasion de son anniversaire, « en mémoire de nos luttes communes ${ }^{19}$. Comme $N$ eesen I'a fait remarquer dans son excellent ouvrage sur Kafka et Brentano:

16. Utitz, 1954, cité par Wagenbach, 1958, p. 107.

17. Brod, 1960, p. 260, cité par N eesen, 1972, p. 28.

18. L'ouvrage était celui de Busse, 1903, une étude de psychophysique (principalement un aperçu de la manière dont était traitée à l'époque la relation entre le corps et l'esprit).

19. Wagenbach, 1958, p. 216, note 426. Berta Fanta créa dans sa demeure un salon régulier des intellectuels praguois, qui était fréquenté par plusieurs des membres du Cercle du Louvre. Les invités des réunions de Fanta ne comptaient pas que Kafka, Bergmann, O skar K raus et Ehrenfels, mais aussi A lbert Einstein, qui fut un temps professeur de physiqueà Prague, Phillipe Frank, un autre physicien qui fut par la suite membre du Cercle de Vienne, et le mathématicien Gerhard K owalewski, qui décrivit les soirées de Fanta dans son autobiographie (1950). O n ne discutait pas que de sujets philosophiques; Brod nous dit, par exemple, qu'au 
« II serait inexplicable que K afka ait participé pendant longtemps aux réunions d'un cercle de philosophes dont le thème ne lui était d'aucun intérêt ${ }^{20}$

$\mathrm{N}$ eesen, et avant lui le biographe de Kafka, Klaus Wagenbach, ont essayé de montrer que non seulement la psychologie de Brentano mais aussi son éthique contribuèrent un peu à déterminer la forme et le contenu de l'œuvre littéraire de Kafka ; que par le biais de sa participation aux soirées du Louvre, Kafka acquit des moyens qui contribuèrent au développement du mode particulier de description de la conscience qui est un trait typique de ses expériences littéraires, et que la psychologie philosophique sous-tendant ce procédé de représentation del'expérience consciente est une interprétation de la psychologie descriptive de Brentano ${ }^{21}$.

Le détail de leur argument fut soumis à un examen critique minutieux, particulièrement par $\mathrm{H}$ armut Binder, qui révèle que même en ces points où les écrits de Kafka suggèrent une affinité conceptuelle ou de terminologie avec les idées brentaniennes, les écarts avec Brentano sont souvent plus significatifs que les similitudes. Binder soutient qu'on peut plus facilement rendre compte de nombre d'aspects du style d'écriture et de pensée de Kafka, qui semblent admettre une explication se référant à une influence brentanienne, en se basant sur des considérations tout à fait indépendantes de la part de $K$ afka22. Ce qui n'implique toutefois pas que l'interprétation de Wagenbach$\mathrm{N}$ eesen soit vide de substance. Binder est le tout premier à être prêt à accepter l'évidence biographique d'un contact plus ou moins soutenu entre Kafka et les brentaniens, et il semblerait donc profitable de prêter attention aux affinités que l'on peut trouver entre lui et Brentano. Q ui plus est, K afka et Brentano sont, comme nous l'avons déjà vu, des représentants d'un vaste mouvement de pensée de l'A utriche de la fin de siècle. Ils révèlent un subjectivisme commun, un intérêt pour la vie intérieure du sujet individuel, aux

cours des soirées de tout un hiver, un certain M. H opf, aussi professeur à Prague et ami d'Einstein, donna un cours combinant un aperçu de la théorie de la relativité et une introduction à la psychanalyse freudienne (Wagenbach, 1958, p. 174).

20. N eesen, 1972, p. 34.

21. L'interprétation de Wagenbach-N eesen fut aussi défendue par Harder, 1962. D'autres travaux sur Kafka et la philosophie européenne, par exemple ceux de Emrich, Bense, Demetz et Pondrom, dont les titres des ouvrages sont donnés en bibliographie, ignorent presque totalement l'arrière-plan brentanien particulier à Prague et son rapport très particulier à Kafka. Emrich et Bense se contentent de quelques parallèles entre la pensée de $K$ afka et la Fundamentalontologie heideggerienne, et l'essai de Demetz est une présentation intéressante mais bien trop brève de l'arrière-plan judaïque typique des intellectuels de la Bohème tels que $\mathrm{H}$ usserl, Freud et Kafka. M ême Pondrom, qui souligne nombre de parallèles intéressants entre Kafka et le meilleur des étudiants de Brentano, Edmund H usserl, parallèles se centrant sur la notion de faire procès au monde, passe complètement à côté de la relation entre Kafka et Brentano. Sur la pensée de Ehrenfels et son influence, voir Fabian, dir., 1986 et A sh, 1995. Sur l'influence de Brentano en général, voir Smith 1994. Sur Brentano et Freud, voir H eaton 1981.

22. Binder, 1966, p. 56-91, vol. I, p. 287. 
dépens des aspects de la réal ité humaine qui ont leurs fondements dans la vie extérieure de la société. Et alors qu'il serait faux de voir en K afka l'interprète d'un simplebrentanisme, on peut montrer que la conception de la conscience qui sous-tend ses écrits repose certainement en partie sur des thèmes brentaniens, même ceux-ci sont modifiés, comme nous le verrons, par une sorte de scepticisme qui s'avère en définitive étranger à la philosophie de Brentano. Toutefois, avec la publication du livre de $\mathrm{H}$ eidsieck, qui offre une richesse de détails textuels et biographiques additionnels supportant l'argument d'origine de Wagenbach-N eesen, un scepticisme de la sorte de celui évincé par Binder essuie un coup sérieux.

\section{L'évidence de la perception intérieure}

Il sera donc nécessaire, pour comprendre l'approche kafkaïenne de la conscience, d'examiner au moins quelques détails de la psychologie théorique de Brentano. Brentano commence par avancer une distinction fondamentale entre deux sortes de phénomènes qu'il appelle respectivement phénomènes physiques et phénomènes psychiques. Des exemples de phénomènes physiques seraient « une couleur, une forme, un paysage que jevois, un accord que j'entends, la chaleur, le froid, une odeur que je sens, ainsi que des formations similaires apparaissant dans l'imaginaire ${ }^{23}$

Les phénomènes psychiques ou mentaux sont simplement, par contraste, nos actes mentaux eux-mêmes :

I'audition d'un son, la vision d'un objet coloré, la sensation du chaud et du froid, ainsi que des états similaires de l'imaginaire [...] la pensée d'un concept général (s'il y a véritablement une telle chose) [...], chaque jugement, chaque souvenir, chaque espérance, chaque inférence, chaque conviction ou croyance, la colère, l'amour, la haine, le désir, un acte de volonté, une intention, l'étonnement, l'admiration, le dédain, et ainsi de suite.

Brentano distingue encore la perception intérieure de la perception extérieure, en référence à leurs objets : les actes de perception intérieure sont les perceptions qui ont des phénomènes psychiques pour objets, les actes de perception extérieure ceux qui ont des phénomènes physiques pour objets. $M$ ais cela laisse obscur ce que « percevoir » un phénomène psychique peut précisément vouloir dire. Brentano n'entendait pas par là, comme nous le montrerons plus tard, l'introspection de ses propres états intérieurs de conscience. II désirait plutôt attirer l'attention sur le fait que chaque état conscient est associé à une conscience de soi, qu'un phénomène ou un état mental qui ne remplirait pas cette condition ne serait pas du tout un « état conscient ». Cen'est pas comme si cet élément de conscience de soi était conçu comme un acte supplémentaire qui existerait d'une façon quel conque dans l'esprit à côté de la conscience extérieure première (ce que la terminologie brentanienne de

23. Brentano, 1924, vol. I, p. 111 et suivantes (p. 79 et suivantes de l'édition anglaise). 
perception « intérieure » et « extérieure » peut malencontreusement suggérer). C'est plutôt un moment ou une partie constituante de la conscience première seulement abstraitement isolable, un moment ne pouvant par nécessité qu'exister enchâssé dans un plus grand ensemble le circonscrivant ${ }^{24}$. Le moment-acte de la perception intérieure peut être conçu comme un acte de vivre-au-travers son objet (le phénomène psychique correspondant) sans aucun objet-cible du type de celui que possèdent typiquement les actes de perceptions extérieures et (si ceux-ci existent) d'introspection ${ }^{25}$.

La thèse brentanienne de la primauté de la perception intérieure a pour conséquence que la vie intérieure, la perception intérieure de phénomènes psychiques peut seule apporter une connaissance certaine. Les seuls objets dont nous pouvons avoir une appréhension absolument certaine sont, tel qu'il le conçoit, seulement ceux dont nous pouvons affirmer d'un savoir absolument évident qu'ils sont en réalité tels qu'ils apparaissent à la conscience. Ceci a pour conséquence que nos perceptions extérieures, c'est-à-dire nos expériences des phénomènes physiques, peuvent toujours être trompeuses. Cette thèse représente une forme de cartésianisme en ceci quel'argument brentanien sur le doute que suscitent les perceptions extérieures repose sur le même genre d'intuition que la discussion de l'illusion perceptuelle de Descartes dans les M éditations : les revendications brentaniennes d'une perception intérieure, source d'une connaissance absolument certaine, rappellent celles de D escartes à propos de ces vérités imperméables au doute méthodique qu'il croit avoir isolées ${ }^{26}$.

Ainsi, quand nous percevons des phénomènes extérieurs, nous ne pouvons savoir avec une évidence absolue que l'objet de nos perceptions est tel qu'il le paraît (nous pourrions, par exemple, être victime d'une hallucination), alors que nous pouvons savoir avec une évidence absolue que tel ou tel état particulier de conscience existe et qu'il est structuré de telle ou telle façon. Brentano prétend qu'illusions et hallucinations sont étrangères au monde de la perception intérieure quand on a bien compris cela.

Ce qu'il entend par cela peut, je pense, être expliqué ainsi : on obtient une évidence absolue seulement si un jugement et ce qui est jugé, c'est-à-dire, pour Brentano, l'objet dont l'existence est affirmée, sont d'une manière ou d'une autre unis en une seule to talité accessible à la conscience, de façon à ce que la rectitude du jugement puisse être directement saisie. Une telle unité est impossible pour les jugements de la perception extérieure, puisque ici les

24. La théorie de ces moments ou de ces parties dépendantes est présentée en détail dans Smith et M ulligan, 1981. Elle fut exposée pour la première fois par $\mathrm{H}$ usserl dans ses premiers écrits sur la logique et l'ontologie formelle, et forme la base indispensable de ses écrits suivants, particulièrement ceux qui se rapportent à la phénoménologie du temps et de la perception sensorielle.

25. Cette interprétation de la théorie de Brentano sur la perception interne est esquissée par Küng, 1978.

26. Cf. Küng, 1978. 
objets visés sont donnés comme transcendant l'acte de juger lui-même. Considérez toutefois des jugements réflexifs de conscience de soi tels que « Je pense » ou « J'ai une image visuelle d'une surface rouge ». De tels contenus de jugements ne sont, comme nous I'avons vu, que de simples moments abstraitement isolables d'actes-totaux qui les comprennent (I'acte de penser, d'avoir telle et telle image visuelle, etc.). A insi, le type d'unité désirée entre le jugement et ce qui est jugé est pour ainsi dire déjà à portée de la main : nos expériences des phénomènes psychiques sont déjà d'elles-mêmes des expériences ayant la qualité d'une évidence absolue. O n peut être trompé en supposant qu'il y a un motif de rouge et de vert (cette expérience comme totalité constituant un objet de la perception intérieure dont l'existence est donnée comme absolue dans l'expérience elle-même) ; le concept d'illusion ne peut avoir pied ici. ${ }^{27}$

Ce qui nous concerne en premier lieu, ce sont les conséquences de la position de Brentano sur notre compréhension du monde ex térieur. II est très important de noter que Brentano lui-même ne nie pas que les objets de la perception extérieure existent en dehors de notre conscience, pas plus qu'il ne nie qu'ils sont tels qu'ils nous apparaissent. II soutient seulement que nous n'avons pas d'évidence inconditionnelle qu'ils existent ainsi. Le monde extérieur, dont notreappréhension est construite (par exemple, linguistiquement) sur la base de l'appréhension de myriades de phénomènes physiques, est réduit à quelque chose qui est seulement probable, comparé à la certitude absolue de l'existence des objets de la perception intérieure. La pensée de Brentano est caractérisée par un réalisme sobre en ce qui concerne le monde de l'expérience, et il s'abstient ainsi de tirer de sa doctrine de la perception intérieure quelque conséquence explicitement sceptique : Brentano se sentait tout à fait à l'aise dans le monde où il croyait vivre ${ }^{28}$. $M$ ais pour quiconque ne serait pas solidement ancré dans la réalité ordinaire et qui aurait été en contact avec la philosophie brentanienne, le glissement du réalisme à une forme ou une autre d'idéalisme ou de scepticisme est aisé à faire, et le sceptique brentanien trouvera qu'il peut, tel Descartes, sanctionner des modes d'expérience où la réalité extérieure projetée par la conscience est troublée ou malicieusement déformée à la lumière des normes d'exactitude assumées par notre expérience de tous les jours. Comme nous le verrons en détail par la suite, ces modes d'expérience différents, et la singulière plasticité du monde qui leur correspond, constituent un thème récurrent dans les écrits de Kafka.

27. Une interprétation utile des arguments de Brentano mentionnés ici est donnée par Chisholm, 1981 ; cf. en particulier sa discussion de la certitude empirique (qui correspond en gros à ce que nous avons appelé ici « évidence absolue »).

28. Cf. Katkov, 1877. 
Q uand R egor Samsa se lève un matin, il ne se sent pas seulement comme un insecte nuisible, il est un insecte nuisible : autrement dit, tout dans son monde extérieur appuie sa conviction que telle est sa nouvelle forme. Au monde extérieur normalement admis est substitué un monde très différent possédant des qualités particulières. Au même moment toutefois, la réalité intérieure de Samsa/Kafka présente des formes et des lignes directrices solides qui passent généralement inaperçues. Du point de vue de nos normes admises concernant la réalité extérieure, une brèche menace bien sûr de s'ouvrir dans la structure de l'expérience de Samsa, une brèche due au fait que le mode d'apparence des objets du (de son) monde extérieur, y compris celui de son propre corps physique, ne s'imbrique pas dans la réalité qui semble continuellement menacer de resurgir chaquefois qu'il a des rapports avec elle (avec ce qu'il pense être la réal ité ex térieure). L e sentiment de cette brèche est toutefois évité par le déplacement continuel de réinterprétations des données qui y parviennent, réinterprétations qui sont le fait de Samsa.

Du point de vue du sceptique brentanien, une brèche de ce type apparaît dans les expériences de n'importe quel individu ; pour la plupart d'entre nous, cette brèche semble être solidement et, comme nous le supposons, correctement mise à l'écart une fois pour toutes, sinon nous adoptons des postulats naturalistes sur la réalité extérieure. A dopter ces postulats consiste en ceci qu'au moins en principe, chaque donnée émanant du monde extérieur aura la priorité sur nos attentes intérieures, sur les rapports entre contenus mentaux dont le motif dérive exclusivement de facteurs internes.

II est bien entendu extrêmement difficile de donner une description claire de cette « brèche », puisque les traits caractéristiques du réel et du subjectif y ont été permutés : pour Brentano, seul le monde intérieur a véritablement un caractère de réalité ; le monde extérieur, que nous prenons habituellement comme norme de réalité, a perdu toutes ses prétentions à un caractère d'absolu (même si, en même temps, on ne nie pas qu'il existe).

Kafka semble toutefois avoir réussi, au moins en partie, à représenter cette brèche de manière littéraire. II ne fait pas directement appel à quelque procédé d'introspection ou à l'observation directe d'expériences mentales. L'idée même d'un tel procédé est, dans la pensée de Brentano, trompeuse. L'observation ou l'attention, processus mentaux impliquant que nous dirigions délibérément notre attention vers un objet, peuvent bien entendu prendre place dans le domaine de la perception extérieure. M ais Brentano soutient que l'observation directe et attentive de sa propre colère, par exemple, ou de tout autre phénomène psychique est impossible puisque, bien entendu, dès que notre attention se serait tournée vers de tels phénomènes, dès que nous serions véritablement en train de vivre notre regard d'observateur, les phénomènes originaux auraient disparu : nous ne serions plus convenablement fâchés ou craintifs mais plutôt attentifs. 
Cette tentative d'introspection commence donc vraiment à ressembler à celle d'un chien qui cherche à attraper sa propre queue. Chaque fois que nous supposons que nos propres états psychiques sont devenus les objets de notre attention, que nous avons réussi dans notre effort de les observer, nous sommes, prétend Brentano, victimes d'une illusion provenant de ce que nous avons par erreur supposé que les phénomènes psychiques peuvent jouer le rôle d'objets qui se tiendraient tranquilles, comme si cela se pouvait, alors que notre attention se concentrerait sur eux.

Kafka était aussi conscient de l'impossibilité d'une observation de soi qui ne serait pas illusoire, bien qu'il l'associe à un scepticisme et à un détachement du monde clairement étrangers à la pensée de Brentano :

Combien misérable est ma connaissance de moi quand elle est comparée, disons, à la connaissance de ma chambre [...]. Et pourquoi? II n'y a pas d'observation du monde intérieur telle que celle du monde extérieur. La psychologie descriptive est en somme probablement au moins un anthropomorphisme, la recherche d'une limite. Le monde intérieur ne peut être que vécu, il ne peut être décrit. La psychologie est la description du reflet du monde terrestre à la surface des cieux, ou plus exactement, la description du reflet que nous imaginons de nous-mêmes, nous qui sommes complètement absorbés par la terre, car il n'y a point de reflet, nous ne voyons que la terre où que nous regardions. ${ }^{29}$

Et de nouveau, dans son journal du 9 décembre 1913 :

II n'est pas possible de noter et d'évaluer toutes les circonstances qui influencent l'humeur du moment, même celles qui sont au travail à travers elle, et qui finalement sont au travail dans l'évaluation, d'où le fait qu'il est faux de dire que je me sentais décidé hier, que je suis au désespoir aujourd'hui. De telles différences prouvent seulement que l'on désire s'influencer soi-même, éloigné le plus loin possible de soi, caché derrière des préjugés et des fantaisies, pour créer temporairement une vie artificielle, comme quelquefois quelqu'un dans un coin d'une taverne, suffisamment dissimulé derrière un petit verre de whisky, entièrement seul avec lui-même, qui ne s'entretient qu'avec de fausses et improbables images ou rêveries.

La perception intérieure doit donc être résolument distinguée de toute forme d'introspection.

Pour comprendre la pertinence de ceci, rappelons la définition que donne Brentano de la perception intérieure comme conscience de phénomènes psychiques. II répartit de tels phénomènes en trois classes qui prétendent épuiser toutes les expériences mentales : les représentations, les jugements et les phénomènes d'amour et de haine ${ }^{30}$. Ainsi, les phénomènes psychiques sont soit des représentations de paysages, de nuances de couleur et de ton, etc., soit des jugements (que tel ou tel objet existe), soit des phénomènes

29. Kafka, 1953, p. 72 (traduction originale de l'auteur que nous avons traduite une nouvelle fois ici). Comparer H eidsieck, 1994, p. 64.

30. Brentano, 1924, vol. I, tome II, ch. 6 . 
d'amour et de haine (catégorie qui comprend, pour Brentano, toutes les émotions et sensations, y compris les sensations de volonté), ou une combinaison des trois. C'est la catégorie des jugements qui illustre peut-être le plus clairement ce qui est impliqué dans la perception intérieure. En jugeant quelque chose, par exemple que cette figure rouge et verte existe, nous avons devant nous, en premier lieu, certains phénomènes physiques, les objets de notre perception extérieure (dans ce cas-ci, les données « rouge » et « vert » dans notre champ visuel). M ais nous avons alors aussi connaissance de ce qui se passe dans notre esprit ; nous sommes conscients - obliquement - du jugement lui-même comme d'un phénomène psychique, comme de quel quechose de vécu-au-travers qui peut être plus ou moins délibéré, plus ou moins appropriéà son contexte, plus ou moins garanti par l'évidence, et ainsi de suite. De la même façon, en voyant directement devant nous la figure elle-même, nous sommes conscients, obliquement, de notre vision de celle-ci. Et c'est cette conscience oblique, présente dans toutes nos expériences mentales quelles qu'elles soient, qui est ce que Brentano entend par perception intérieure.

Parce que les perceptions intérieures sont, de par leur nature, des moments dépendants de totalités d'actes plus inclusifs qui comprennent aussi des moments de perception extérieure, et parce que la conscience oblique d'un acte mental présuppose aussi qu'il y a une quelconque conscience directe de l'objet del'acte, il s'ensuit qu'il est impossible de communiquer les données de la perception intérieure, excepté en association avec une structure adéquate de perception extérieure. $\mathrm{N}$ ous voudrions d'abord, pour une telle structure, en appeler seulement à l'expérience ordinaire, mais étant donné les tendances et les habitudes avérées del'esprit, cela placerait des obstacles presque insurmontables dans notre voie de restitution des données de la vie intérieure, puisqu'en de telles circonstances les attentions du lecteur suivraient leur cours naturel (elles seraient dirigées vers le déroulement externe de I'action). M ais il semblerait que les formes et les structures particulières de la conscience intérieure pourraient être rendues accessibles, être mises en repré sentation, si l'esprit du lecteur pouvait être d'une manière ou d'une autre distrait de son intérêt pour ce qui se déroule dans un monde extérieur figuré, de telle façon qu'il ne soit pas dirigé vers la représentation de simples et futiles actes d'introspection. Je crois que ceci peut être réalisé si l'ordre attendu du monde extérieur est présenté de manière brute, pas à pas, mais d'une certaine manière interrompu.

Considérez par exemple dans le passage suivant la manière dont notre attention s'enchâsse dans la vie intérieure du narrateur :

Je marchais, imperturbable. $M$ ais puisque, en tant que piéton, je craignais l'effort de gravir le chemin de montagne, je le flattais peu à peu, le laissais s'incliner vers une distante allée. Les pierres s'évanouissaient à volonté et le vent disparut.

Je marchais d'un pas brusque et alors que je descendais je levai la tête, raidis mon corps et croisai mes bras derrière ma tête. Parce que j'aimais les forêts de 
pins, je passai par des bois de la sorte, et parce que j'aimais regarder silencieusement les étoiles, les étoiles apparurent lentement dans le ciel, comme de coutume. Je ne vis que quelques nuages moutonnés qu'un vent soufflant juste à leur hauteur tirait dans l'air, à l'étonnement du piéton.

À l'opposé et à quelque distance de mon chemin, probablement séparée de lui par une rivière, je fis jaillir une énorme montagne dont le plateau, recouvert de broussailles, jouxtait le ciel. Je pouvais voir très nettement les petits rameaux des plus hautes branches et leurs mouvements. Cette vue, ordinaire comme elle peut l'être, me rendit si heureux que j'en oubliai, tel un petit oiseau sur un branchage de ces broussailles, de faire se lever la Lune. Elle se trouvait déjà derrière la montagne, sans doute irritée par cette attente. ${ }^{31}$

Le mode narratif des écrits de Kafka est, à un point frappant, la description d'une expérience par son acteur principal. M ais Kafka ne recourt pratiquement jamais à l'expression d'une introspection active. La description d'une conscience oblique du monde intérieur est plutôt réalisée par contraste avec l'ordre typique ou attendu de la réalité extérieure, par une césure de l'imbrication normale entre l'intérieur et l'extérieur. Cette qualité de littéralité obstinée des écrits de Kafka semble être le moyen de prendre au dépourvu le lecteur quand son attente d'un ordre naturel ou raisonnable du monde extérieur à laquelle elle conduit est bouleversée. Les descriptions que fait Kafka d'une réalité dépouillée ne sont jamais superflues, ne sont jamais introduites, par exemple, à des fins d'ornement. M ais il ne se donne pas non plus grand mal pour parvenir à un réalisme psychologique ou social particulier dans ses tableaux, surtout en ce qui concerne ses personnages secondaires. La description de la réalité extérieure sert plutôt une fin prédominante qui est de permettre l'exposition de quelque aspect particulier de la conscience oblique.

\section{De notre connaissance du bien et du mal}

La réputation de Brentano en A utriche durant les premières décennies de notre siècle, en dehors du petit cercle des philosophes et psychologues théoriques, était principalement celle d'un éthicien. Son ouvrage Vom U rsprung sittlicher Erkenntnis (De l'origine de la connaissance morale) était aussi familier à Vienne que l'étaient, par exemple, les Principia E thica de M oore (qui, d'une certaine manière, lui ressemblent) ${ }^{32}$ pour les intellectuels de Bloomsbury à Cambridge. Cette popularité était en partie due au fait que

31. «Beschreibung eines Kampfes», Kafka, 1970, p. 207 et suivantes (traduction anglaise de 1971, p. 22).

32. La préface des Principia Ethica de M oore contient le passage suivant : « When this book had been already completed, I found, in Brentano's "O rigin of our Knowledge of Right and Wrong", opinions far more closely resembling my own, than those of any other writer with whom I am acquainted. »C onsulter aussi le compte-rendu de M oore sur les travaux de Brentano, publié en 1903. 
Brentano avait réussi dans cet ouvrage à capter l'essentiel de la vision du monde partagée par les intellectuels centre-européens de son époque, et il serait donc surprenant que nous ne trouvions pas quelques échos de la pensée éthique de Brentano dans les écrits de $K$ afka ${ }^{33}$.

Comme on l'a déjà fait remarquer, trois catégories fondamentales de phénomènes psychiques sont reconnues par Brentano : les représentations, les jugements et les phénomènes d'amour et de haine. Chaque action consciente est accompagnée ou fondue dans un ou plusieurs de ces phénomènes : par exemple, l'action de décapiter peut être accompagnée chez le bourreau de la perception intérieure de son propre dégoût. On supposera maintenant aisément que c'est à la dernière de ces catégories que se rapportent les questions éthiques. Ainsi, les critères proposés par les philosophes pour la détermination d'une conduite éthiquement correcte ou incorrecte tendirent à consister en affirmations assurant que la rectitude éthique devait être déterminée à la lumière de certaines considérations relatives aux sentiments et aux émotions : I'action qui est bonne est l'action qui est sentie bonne, ou qui est accompagnée de bonne volonté, ou qui participe au bonheur, etc.

La plus importante et, aux yeux de Brentano, la plus fallacieuse de ces doctrines est celle de l'utilitarisme, qui affirme que la justesse ou la fausseté éthique d'une action est mesurée directement selon le plaisir ou la douleur qu'elle engendre. Cette conception de l'éthique est du même acabit que les doctrines du naturalisme positiviste devenues prédominantes parmi les philosophes du XIX ${ }^{\mathrm{e}}$ siècle, qui se fondaient sur le rejet de la possibilité d'une évidence apodictique dans la sphère de l'éthique, comme dans toutes les autres sphères, d'ailleurs. H usserl et les néo-kantiens mirent fin, au tournant du siècle, à la prédominance du positivisme dans le monde germanophone mais son premier défi sérieux fut la doctrine brentanienne du caractère fondamental du jugement pour l'éthique. La conviction de Brentano quant à la possibilité d'une évidence apodictique provient de sa conception des implications de l'existence de certaines variations dans la qualité de l'évidence en général, et donc aussi dans la qualité des actes mentaux sur l'évidence. Considérez, par exemple, relativement à la catégorie la plus simple de phénomènes psychiques (la catégorie des représentations), le fait que nous puissions percevoir un paysage plus ou moins clairement et que notre perception puisse avoir un plus ou moins grand degré de contenu intuitif ; ou le fait que nous puissions être conscients de nous rappeler le visage ou le numéro de téléphone d'une connaissance plus ou moins distinctement ; ou le fait que

33. Unefois encore, cependant, la possibilité d'une influence directe ne doit pas être sousestimée. Wagenbach, en effet, voit les idées de Brentano comme ayant eu une influence très précise sur les écrits suivants de Kafka : "L'influence du cercle de Brentano [...] peut difficilement être sous-estimée, en particulier en tant qu'élément du développement de son rigorisme éthique tardif fondé sur une sorte de nécromancie du jugement (U rteilsmagie) », 1958, p. 116. 
nous puissions imaginer le futur ameublement d'une pièce vide avec plus ou moins d'acuité ; et ainsi de suite. Chacun de ces trois types de représentations présente des variations claires et indéniables dans la qualité de l'évidence associée. Alors que ces variations peuvent dépendre en partie d'une aptitude innée (une bonne vue, par exemple), elles peuvent aussi dépendre d'un entraînement, qui peut à son tour être encouragé par des conventions morales et des arrangements institutionnels particuliers à la société.

Ces variations dans la qualité de l'évidence sont également un fait bien connu dans la sphère du jugement. Ainsi, d'une part, nous pouvons être conscients qu'une croyance spécifique, qui fut la nôtre pendant plusieurs années, reposait simplement sur l'autorité ou la coutume, sur une information mal vérifiée, ou sur une émotion ou un sentiment dont les origines n'ont jamais été explicitement considérées. D'autre part, nous pouvons être conscients qu'une croyance repose sur des données factuelles que nous avons soumises aux tests les plus rigoureux, ou qu'elle repose sur l'évidence immédiate (l'évidence de la perception intérieure), soit immédiatement, soit par le biais d'étapes déductives qui elles-mêmes reposent sur une évidence immédiate. L'idée de certitude absolue, de la plus grande évidence immédiate possible d'un jugement - qui n'a rien à voir avec les sentiments de certitude qui peuvent l'accompagner - s'obtient en considérant, sur la base d'un examen de tous les axes de variation possibles dans la qualité de l'évidence, le type de jugement qui présenterait, dans les circonstances favorables requises, l'évidence maximale concevable sur un tel axe.

Brentano veut défendre la thèse qu'un jugement a pour qualité d'être susceptible d'évidence absolue. II soutient que cette thèse relève parfaitement du sens commun : il n'y a rien, dans notre expérience ordinaire de tous les jours ou dans la pratique du scientifique, qui puisse raisonnablement nous conduire à douter qu'un tel caractère soit quelque chose d'objectif. Ainsi, bien que quelques-uns des axes de variations que nous avons considérés reposent sur la présence ou l'absence d'éléments subjectifs (par exemple, la force de l'engagement émotionnel), cette présence ou cette absence d'éléments subjectifs n'est pas à son tour quelque chose d'objectif. La qualité de certitude est objective en cela qu'elle ne repose pas sur la répartition particulière, à l'intérieur d'une population donnée, de dispositions à contrôler ses propres prémisses, à examiner des arguments, etc., non plus que sur les techniques de construction d'une théorie etc., dans l'existence à n'importe quel stade donné. Q u'un jugement ait un pouvoir d'évidence ou de certitude absolue est quelque chose qui relève seulement du jugement et qui resterait vrai même si une intuition correspondante de la part du sujet jugeant ne se réalisait jamais.

Une autre manière d'exprimer le fait qu'un jugement a en lui-même le pouvoir d'être exercé avec une évidence absolue est de dire que le jugement est vrai. Pour Brentano bien sûr, les seuls jugements dont le caractère d'évidence absolue peut être saisi par des sujets pensants tels que les êtres humains 
sont les jugements relatifs aux phénomènes psychiques - ce qui n'implique pas qu'ils soient les seuls jugements à être vrais ${ }^{34}$.

II y a une analogie dans la sphère de l'éthique des jugements de faits : les jugements defaits peuvent être exercés avec un plus ou moins grand degré d'honnêteté, etc., et les phénomènes d'amour et de haine, d'approbation et de désapprobation, peuvent, dans une certaine mesure, être troublés par des éléments extérieurs ou des éléments qui entrent en conflit avec ces derniers. A insi, nous pouvons cerner un analogue éthique à la notion d'évidence absolue - l'évidence d'un acte d'amour, par exemple, accompli de manière à participer de la plus grande clarté, immédiateté, etc., possibles. Q ue, dans les faits, des actes présentent toujours cette particularité n'est pas ici la question : nous avons seulement le souci établir la cohérence de cette notion et la consistance du tableau du monde éthique qu'elle présente. L'analogue éthique de la vérité est, en ce cas, la qualité de justesse ainsi déterminée : une action est éthiquement correcte si elle repose, ou pourrait en principe reposer, sur des actes d'amour ou d'approbation participant à un caractère établi de pureté maximale. Et c'est la connaissance de la justesse ou de la fausseté éthique, le jugement éthique, et non l'amour ou la haine, le plaisir ou le déplaisir, qui forme le fondement de l'éthique brentanienne.

Le champ des actions dont le caractère évident de bonté est donné de manière immédiate par un tel critère de justesse peut en fait être très limité (il serait confiné au genre d'exemple suivant : parvenir à la connaissance de la vérité, encourager l'amitié, faire le mieux le plus largement possible). II n'est pas possible de fabriquer de paire de lunettes éthique qui nous permettrait de saisir immédiatement le degré de justesse éthique d'une action dans les circonstances hautement complexes au sein desquelles se posent normalement les problèmes éthiques. Comment alors, si c'est possible, faire en sorte que l'éthique brentanienne s'occupe non pas seulement de simples truismes mais plutôt de véritables dilemmes éthiques dans la réalité quotidienne?

$N$ otons en premier lieu que le statut des problèmes éthiques complexes n'est pas, en principe, différent de celui des problèmes factuels ayant un même de degré de complexité. Cen'est qu'avec un relativement petit nombre de jugements scientifiques qu'on parvient à une évidence véritablement adéquate : I'application quotidienne de la science au monde matériel repose sur le développement de pratiques coutumières qui ne se fondent que partiellement sur cette évidence. L'utilité de ces expédients masque le fait que, dans le développement de notre connaissance du monde matériel, il y a, à n'importe quelle étape, un seuil au delà duquel aucune solution certaine ne peut être apportée à des jugements. Et Brentano soutient que ce qui s'applique à la sphère des jugements de faits s'applique aussi à la sphère de l'éthi-

34. De l'hypothèse que seuls les jugements relatifs aux phénomènes psychiques sont vrais, il s'ensuivrait, bien entendu, que le monde réel tel que nous le concevons normalement n'existe tout simplement pas. 
que. Dans cette dernière aussi, le besoin continuel de solutions à des problèmes complexes (tels que ceux qu'affrontent, par exemple, juges ou politiciens) mena à des réseaux complexes de procédés empiriques à différents niveaux ainsi qu'au retranchement progressif des coutumes et conventions qui y sont associées, de telle manière que l'architecture du droit dans son ensemble peut être considérée, d'un certain point de vue, comme un instrument de résolution des dilemmes éthiques de la société évoluant très lentement. Le fait que de tels expédients doivent être acceptés dans la sphère de la praxis ne doit pas impliquer qu'il est injustifié de considérer la manière dont le seuil de l'évidence peut en principe être étendu. Et cela ne sanctionne pas davantage l'affirmation selon laquelle la résolution des problèmes éthiques est - comme le croient les positivistes - simplement objet de convention. La théorie éthique de Brentano est, plus que tout autre chose, I'affirmation de l'objectivité de la valeur éthique et de l'existence (qu'on ne peut peut-être pas découvrir factuellement) de solutions intrinsèquement optimales à tous les dilemmes éthiques. De la même manière qu'une proposition est en ellemême vraie ou fausse indépendamment de notre reconnaissance de sa valeur de vérité, une action est éthiquement correcte ou incorrecte indépendamment de notre appréhension de sa valeur morale.

\section{$5 \quad$ Le tribunal intérieur}

N ous pouvons maintenant déterminer plus précisément le rôle joué par le jugement, et plus spécifiquement par le jugement qui a caractère d'évidence, dans la sphère de l'éthique. Une action est éthiquement correcte si elle peut en principe reposer sur des actes d'amour ou d'approbation qui participent à un caractère de pureté maximale. M ême là où il est impossible d'établir un lien direct entre une action visant un objet aimé et un jugement de la justesse del'acte d'amour qui la sous-tend, il doit pourtant être possible de vérifier la justesse de cette action par des moyens indirects, à savoir par des chaînes de jugements (qui ont un caractère d'évidence et qui se rapportent aux phénomènes psychiques du sujet impliqué) soumises aux critères suffisants de témoignage, de clarté, etc., qui ont été établis ci-dessus. M ais alors, les processus impliqués dans l'attestation de la justesse de nos actions, dans le fait de rendre un jugement sur soi-même (processus comprenant la réunion et le classement de données pertinentes de la perception intérieure, la mise à l'épreuve de conclusions, etc.), transforment la vie intérieure de l'individu éthique en une sorte de tribunal.

N ous pouvons peut-être comprendre l'importance du subjectivisme radical qui sous-tend l'éthique brentanienne en considérant à nouveau la situation sociale et politique de la fin de l'Empire des $\mathrm{H}$ absbourg. A près plusieurs siècles d'un régime autocratique, l'A utriche fit la brève expérience d'une forme d'organisation politique libérale et démocratique, expérience qui se termina abruptement. Son échec fut, paradoxalement, le résultat de 
son succès initial. Elle avait placé un pouvoir (limité) entre les mains de bourgeois libéraux et éclairés qui firent beaucoup pour libérer l'Empire du joug del'absolutisme. Toutefois, avec l'essor de la démocratie, le pouvoir et l'activisme politique passèrent progressivement dans les mains de meneurs de mouvements politiques de masse très peu éclairés qui menaçaient d'éliminer ces conventions de justice et de mesure qui sont précisément les conditions préalables d'un ordresocial démocratique ${ }^{35}$. Le chaos politique qui s'ensuivit s'acheva par le retour du pouvoir en des mains autocratiques.

Cette foi inébranlable dans le pouvoir d'amélioration sociale de la pensée libérale, foi partagée par les intellectuels européens tout au long du XIX siècle, était de ce fait anéantie, de telle façon que cela affecta la vie intellectuelle autrichienne pendant plusieurs décennies ${ }^{36}$. Brentano réagit à l'effondrement du libéralisme en se tournant vers une doctrine éthique abstraite et idéalisée qui renfermait à la fois des éléments libéraux et traditionalistes, mais qui reposait sur des fondements essentiellement individualistes. La génération d'intellectuels autrichiens qui lui succéda, qui ne comprenait pas seulement $K$ afka mais aussi, par exemple, Weininger et $W$ ittgenstein, fit face au dilemme en abandonnant entièrement les prémisses libérales, se détournant de la société en embrassant des doctrines éthiques encore plus individualistes ${ }^{37}$. Ainsi perçoivent-ils le mécanisme de l'ordre social et politique avec l'incompréhension d'un somnambule. Et pourtant, leurs pensées et leurs actions ne se déroulent pas dans un espace éthiquement vide. Elles sont plutôt marquées par une confusion éthique omniprésente (la culpabilité). Là où un sérieux effort est fait pour briser cette confusion, pour substituer quelque lumière à l'obscurité éthique, comme, par-dessus tout, dans Le Procès, on peut le voir comme n'impliquant que ces processus internes qui, dans le système de Brentano, transforment l'esprit de l'individu concerné en ce qui équivaudrait à un tribunal.

L'individu confus dans son éthique ne peut résoudre son dilemme intérieur, ne peut vivre une vie éthiquement satisfaisante, que s'il en arrive au point où il peut se juger lui-même. Ceci n'est possible que s'il connaît la portée de ses moyens physiques et mentaux, que s'il peut entièrement appréhender l'importance de ses propres limites et s'il a la patience de ne pas vouloir les transgresser de manière illusoire ${ }^{38}$.

35. Les mouvements impliqués ici étaient, en dehors des différents nationalismes établis de l'Empire, le pan-germanisme, le socialisme chrétien et, en réponse à ces deux derniers, le sionisme (cf. Schorske, 1967). Les doctrines subséquentes du nazisme sont très clairement issues de mouvements de ce genre.

36. Cf. Schorske, 1980.

37. L'individualisme anti-libéral était souvent associé à une forme de conservatisme paisible auquel se rattachait un respect de la loi et de l'autorité pour eux-mêmes, point de vue qui considérait toute forme de conviction révolutionnaire comme immorale. Cf. Smith, 1978, p. 429 et suivantes.

38. «La connaissance complète de soi-même. Être capable de saisir l'entièreté de ses facultés comme une petite balle. Être capable d'accepter le plus grand déclin comme quelque 


\section{$6 \quad$ Le cas de Joseph $\mathrm{K}$.}

Le récit de Kafka dans L e Procès est à la frontière entre le droit et la psychologie, en exploitant par-dessus tout l'ambiguïté entre lejugement comme acte mental et le jugement comme procédé légal. Cette ambiguïté n'est jamais résolue parce que notre conception commune de la limite entre le monde intérieur et le monde extérieur (social) a été suspendue. Un certain ensemble de phénomènes psychiques, particulièrement ceux qui sont liés au constant besoin de justification de $K$., peuvent être considérés comme déterminant eux-mêmes une réal ité projetée, confondue ou enchâssée dans la réal ité ex térieure ordinaire. De la même manière que la conception qu'a Gregor Samsa de lui-même et de son corps détermine sa morphologie et son comportement dans le « monde extérieur » hybride qu'il a créé, le procès continuel vécu par $K$. à l'intérieur de lui-même est aussi un procès réel dans le monde extérieur où il s'imagine vivre. Puisque ce monde est en partie une création, le procès aussi n'est jamais que partiellement réel (il ne peut être sanctionnéque par les actes mentaux de K. lui-même).

II nous est dit au tout début du texte de K afka que les autorités faisant procès à $K$. ne font pas leur enquête afin de déterminer sa culpabilité. Plutôt, elles ne font, " tel qu'il est écrit dans la loi », que « suivre le chemin de sa culpabilité ». K. est arrêté ; mais on fait bien comprendre que cette arrestation ne doit se mettre en travers ni de sa profession ni de sa vie de tous les jours. Q uand K. cherche le tribunal, il n'a d'abord aucune idée del'endroit où il est sensé aller. $M$ ais il se rappelle soudain la déclaration à l'effet que le tribunal « suivrait le chemin de la culpabilité », et il voit « qu'il s'ensuit immédiatement que le bureau d'enquête doit se trouver au haut de la rampe de l'escalier qu'il a choisi par accident ». Le tribunal est donc là où $K$. lui-même a ordonné qu'il soit, comme s'il le portait avec lui en tant que partie de ses effets personnels. Et alors qu'il s'assied dans le prétoire, il réalise que le jugement lui-même n'est jugement que s'il le reconnaît comme tel. Une défense devant la cour n'est pas vraiment permise, mais seulement tolérée - et il y a même quelques disputes concernant ce point. Et la procédure se fait en géné ral à huis clos, même pour l'accusé. C ar ce ne sont pas les faits réellement pré sentés à la cour qui sont décisifs, mais ce qui a lieu autour, dans la chambre de délibération et dans les corridors voisins. Il y a une chance que l'innocence de l'accusé joue un rôle dans l'acquittement qui pourrait effectivement avoir lieu, bien qu'un acquittement soit bien sûr quelque chose de rare, et qui ne se produit jamais en influençant d'une manière ou d'une autre le juge. Toutefois quand cela arrive, non seulement les mandats de comparution et tous les autres documents du procès doivent être détruits, mais on doit aussi rendre inexistants l'acquittement et le procès lui-même.

chose de familier et de conserver pourtant une certaine souplesse » (Journal du 8 avril 1912). O u, comme le formule N eesen (1972, p. 143) : "Seul dans la sérénité, dans la prise de conscience de ses propres possibilités et avec une patiente modestie, peut-on vivre une vie satisfaisante. » 
À la fin, on demande à $K$. de décider lui-même de reconnaître ou de ne pas reconnaître la cour. « La cour ne veut rien de vous. Elle vous acceptera si vous venez, et ellevous libérera si vous partez. » Et alors queK. est conduit à son supplice, il se sait devoir êtrelui-même son propre bourreau. Q ui donc, se demande $K$., était le juge qu'il n'a jamais vu, et où donc était le tribunal où, jusqu'à la fin, il n'est jamais arrivé? ${ }^{39}$ Le juge, la cour et le procès luimême sont des projections de l'expérience intérieure de K., des projections du monde de la perception intérieure dans le monde des faits extérieurs. Et au moins un aspect du pouvoir agissant derrière cette projection est la conviction de K. qu'une action moralement correcte lui demande de parvenir à un jugement absolument évident. Parce qu'il pense que c'est quelque chose qu'il ne peut atteindre que par un processus de débat, processus qui présupposerait toute une série d'investigations et de discussions et parce que, comme il en est vaguement conscient, il est incapable d'être honnête avec luimême, il crée une série d'événements ayant la nature d'un procès judiciaire, obscurément perçu comme tel, et qui ferait le travail pour lui : «Puisqu'on se clarifie soi-même au moyen d'un jugement, et puisque ce jugement doit avoir lieu au centre du moi, ce moi devient une sorte de tribunal [...] $\gg{ }^{40}$

Là où, comme dans l'A utriche de la fin de l'Empire des $\mathrm{H}$ absbourg, le respect de la société en tant qu'arbitre moral est détruit, ce rôle doit échoir à la conscience du sujet individuel. Là où récompense et punition éthiques ne peuvent plus être considérées comme des événements dans le monde, elles doivent être conçues comme résidant en l'action elle-même.

Culpabilité et punition ne sont pas deux choses distinctes; elles sont une. II est alors possible d'en retirer au moins ce réconfort, qu'il n'est pas de criminel qui échappe vraiment à la punition qu'il mérite. Les fondements philosophiques de ce monde introverti furent décrits par Brentano et, plus radicalement encore, par Weininger et Wittgenstein. $M$ ais c'est $K$ afka qui montra avec le plus d'acuité à quoi il pourrait bien ressembler de vivre dans un tel monde, un monde dans lequel lesujet individuel est à tel point entraîné à l'intérieur de lui-même que la conviction d'un ordre extérieur de la réalité commence lentement à revêtir la forme d'une superstition.

\section{Bibliographie}

Ash, M itchell G., Gestalt Psychology in German Culture 1890-1920: $\mathrm{H}$ olism and the Q uest for O bjectivity, Cambridge, Cambridge University Press, 1995

Bense, M ., Die Theorie Kafkas, Berlin, Kiepen H auer and Witsch, 1952

39. Ce résumé du Procès est tiré de celui de N eesen, 1972, p. 202.

40. I bid., p. 205, et cf. aussi à la même page : « puisque le tribunal est en Joseph K. luimême, il devient compréhensible qu'il n'ait pas à interrompre le cours normal de sa vie. Et pour la même raison il est partout où $\mathrm{K}$. le cherche, ainsi que là où il ne l'attend pas. » 
Bergmann, H., Untersuchungen zum Problem der Evidenz der inneren Wahrnehmung, $\mathrm{H}$ alle, N iemeyer, 1908

_- , D as philosophische Werk Bernard Bolzanos, H alle, N iemeyer, 1909

Bezzel, C., Kafka-Chronik, M ünich, H anser

Binder, H ., M otiv und G estaltung bei Franz Kafka, Bonn, Bouvier, 1966

- - , Kafka-H andbuch, 2 vol., Stuttgart, Kröner, 1980

Brentano, F., Psychologie vom empirischen Standpunkt. $2^{\mathrm{e}}$ édition, avec une introduction de O skar Kraus, Leibniz, M einer, 1924 (1 1 ère édition: 1876) (traduction anglaise: M cAlister, L.I., Psychology from an Empirical Standpoint, London, R outledge, 1973)

- - , Vom U rsprung sittlischer Erkentniss, $3^{\mathrm{e}}$ édition révisée, avec une introduction de Oskar Kraus, Leipzig, M einer, 1934 (1 ${ }^{\text {ère }}$ édition : 1989) (traduction anglaise: Chisholm, R.M ., et Schneewind, E.H., The origin of our Knowledge of Right and Wrong, London, Routledge, 1969)

- - , D eskriptive Psychologie, H amburg, Felix M einer, 1982 (traduction anglaise : M üller, Benito, D escriptive Psychology, London/N ew York, Routledge, 1995)

Brod, M ., Streitbares L eben - Autobiographie, M unich, Kindler, 1960

- - , Franz K afka - Eine biographie, $3^{\mathrm{e}}$ édition, Frankfurt, Fischer, 1974

Busse, L., G eist und Körper - Seele und L eib, Leipzig, Dürr, 1903

Chisholm, R. M ., The First Person, H assocks, H arvester, 1981

Demetz, P., «Kafka, Freud, Husserl: Probleme einer Generation», Z eitschrift für R eligions und G eistesgeschischte, 7, 1955, p. 59-69

Emrich, W., Franz Kafka, $2^{\mathrm{e}}$ édition, Frankfurt, Athenäum, 1960

Fabian, Reinhard, dir., Christian von Ehrenfels: Leben und Werk, A msterdam, Rodopi, 1986

Grassl, Wolfgang et Smith, Barry, «A Theory of Austria », dans N yiri, J. C., dir., From Bolzano to Wittgenstein : The Tradition of A ustrian Philosophy, Vienne, Hölder-Pichler-Tempsky, Dordrecht, Reidel, 1986, p. $11-30$

Haller, R., «Wittgenstein und die wiener Schule», dans Studien zur österreischichen Philosophie, Amsterdam, Rodopi, 1978, p. 163-188 (traduction anglaise dans N yiri, J. C., dir., 1981, p. 91-112)

$\mathrm{H}$ arder, M .-L., M ärchenmotive in der D ichtung Franz Kafkas, dissertation, Fribourg, 1962

H eaton, J. M., «Brentano and Freud », dans Smith, B., dir., Structure and Gestalt - Philosophy and Litterature in Austria-H ungary, A msterdam, Benjamins, 1981, p. 161-194

Johnston, W.M ., The Austrian M ind - Intellectual and Social H istory 18481938, Berkeley, University of California Press, 1972

Kafka, F., H ochzeitsvorbereitung auf dem $L$ ande und andere Prosa aus dem N achlass, N ew York, Schœn, 1953

—- , Sämtliche E rzählungen, Frankfurt, Fischer, 1970 
Katkov, G., "The World in Which Brentano Believed He Lived», dans Chisholm, R. M ., et H aller, R., dir., D ie Philosophie Franz Brentanos, A msterdam, Rodopi, 1978, p. 11-28

$\mathrm{H}$ eidsieck, Arnold, The Intellectual Contexts of Kafka's Fiction : Philosophy, Law, Religion, Columbia, Camden H ouse, 1994

Kowalewski, G., Bestand und Wandel - Meine Lebenserrinerungen zugleich ein Beitrag zur neueren $\mathrm{G}$ eschichte der M athematik, M unich, Oldenbourg, 1950

Küng, G., «The Phenomenological Reduction as Epoché and as Explication », The M onist 59, 1975, p. 63-80

- - , «Zur Erkenntnistheorie von Franz Brentano », dans Chisholm R. M ., et $\mathrm{H}$ aller, R., dir., Die Philosophie Franz Brentanos, Amsterdam, Rodopi, 1978, p. 169-82

M arek, Johannes et Smith, Barry, «Einleitung zu A. M artys 'Elemente der deskriptiven Psychologie'», Conceptus, 21, 1987, p. 33-48 (introduction de l'éditeur aux extraits des conférences de $M$ arty : ibid., p. 4966)

M arty, A., Untersuchungen zur G rundlegung der allgemeinen Grammatik und Sprachphilosophie, vol.1 (seul volume publié), H alle, N iemeyer, 1908

M oore, G. E., Principia Ehtica, Cambridge, University Press, 1903

-- , «R eview of Franz Brentano : The $O$ rigin of the Knowledge of Right and Wrong », International J ournal of Ethics, 14, 1903a, p. 115-123

Neesen, P., Vom Louvrezirkel zum Prozess - Franz Kafka und die Psychologie Franz Brentano, Göppingen, Kümmerle, 1972

$\mathrm{N}$ eurath, $\mathrm{O}$. et al., I nternational Encyclopedia of U nified Science, vol. 1, no1, Chicago, University of Chicago Press, 1938

N yiri, J. C., " Intellectual Foundations of Austrian Liberalism », à paraître dans Smith, B. et Grassl, W., dir., Austrian Philosophy and Sical Thought, M ünich, Philosophia, 1982

- - dir., Austrian Philosophy : Studies and Texts, M unich, Philosophia, 1981

Pondrom, C. M., "Kafka and Phenomenology. Joseph K's Search for Information », Wisconsin Studies in Comtemporary Litterature, 8, 1967, p. 78-95

Schorske, C. E., «Politics and the Psyche in fin de siècle Vienna, Schitzler and H ofmannsthal », A merican H istorical Review, 66, 1960/1961, p. 930946

- - « Politics in a N ew Key : An Austrian Triptych », Journal of M odern $\mathrm{H}$ istory, 39, 1967, p. 343-386

- - , Fin-de-Siècle Vienna, N ew York, Knopf, 1980

Smith, B., "Law and Eschatology in Wittgenstein's Early Thought », Inquiry, 14, 1978, p. 425-441 
- - « Kafka and Brentano : A Study in Descriptive Psychology », in Smith, B., dir., Structure and G estalt - Philosophy and Litterature in A ustriaH ungary, A msterdam, Benjamins, 1981

- - , A ustrian Philosophy: The Legacy of Franz Brentano, La Salle and Chicago, O pen Court, 1994

Smith, B. et M ulligan K., "Pieces of a Theory », à paraître dans Smith, B., dir, Parts and Moments - Studies in Logic and Formal O ntology, M unich, Philosophia, 1982

Utitz, E., « Erinnerungen an Franz Brentano », Wissenschaftliche Zeitschrift der M artin-L uther-U niversität, 4, 1954

Wagenbach, K., Franz Kafka - Eine Biographie seiner Jugend - 18831912, Berne, Francke, 1958

Weininger, 0. ., G eschlecht und Chracter - Eine Prinzipielle U ntersuchung, Vienne, Braumüller, 1903 